\title{
AMALGAMATION OF CONTOUR, TEXTURE, COLOR, EDGE, AND SPATIAL FEATURES FOR EFFICACIOUS IMAGE RETRIEVAL
}

\author{
Anju Maria ${ }^{1}$, Dhanya $\mathbf{S}^{2}$ \\ ${ }^{1}$ M.Tech Student, ${ }^{2}$ Assistant Professor, Electronics and Communication, FISAT, Kerala, India
}

\begin{abstract}
From the past few years, Content based image retrieval (CBIR) has been a progressive and curious research area. Image retrieval is a process of extraction of the set of images from the available image database resembling the query image. Many CBIR techniques have been proposed for relevant image recoveries. However most of them are based on a particular feature extraction like texture based recovery, color based retrieval system etc. Here in this paper we put forward a novel technique for image recovery based on the integration of contour, texture, color, edge, and spatial features. Contourlet decomposition is employed for the extraction of contour features such as energy and standard deviation. Directionality and anisotropy are the properties of contourlet transformation that makes it an efficient technique. After feature extraction of query and database images, similarity measurement techniques such as Squared Euclidian and Manhattan distance were used to obtain the top $N$ image matches. The simulation results in Matlab show that the proposed technique offers a better image retrieval. Satisfactory precision-recall rate is also maintained in this method.
\end{abstract}

Keywords: Contourlet Decomposition, Local Binary Pattern, Squared Euclidian Distance, Manhattan Distance

\section{INTRODUCTION}

Recent days, witnessed a rapid growth of the storage spaces accommodating enormous image databases. Acquiring the appropriate image from the huge collection is tedious. So an efficient browsing, searching and retrieval technique needs to be incorporated. In the budding stages of the retrieval system, text based retrieval was employed. In those systems, images were annotated with the text. Automatic generations of descriptive texts were not viable and hence it requires manual annotation. This pave the way to content based image retrieval (CBIR) system.

CBIR utilizes the visual features of an image. Wavelet and Fourier transformation based CBIR were the widely used techniques. Although they were found acceptable, these techniques could not provide any details about smooth contours. Here an effective system based on contourlet transformation is proposed. This system could extract the contents of the image in terms of directional contours, horizontal and vertical edges of the image.

The previous research work, CBIR using contourlet transform (CT) [1] proposed the relevance of using contourlet transform, as it offered high retrieval rate and less computational complexity. The work on Rotation invariant image description with local binary pattern (LBP) histogram Fourier feature [2] could reveal that the Discrete Fourier transform (DFT) based features derived from Uniform LBP were invariant to rotations.
CBIR using histogram, color and edge [3] examined the retrieval efficiency based on different features like image histogram, color values and edge values. An approach for combining the features of the images was proposed in the work Integrating contourlet features with texture, color and spatial features for image retrieval [4]. In color - Texture based image retrieval system [5] both color and texture features were used to retain accurate retrieval result. Here color histogram for color extraction and pyramid structure wavelet transform model for texture extraction were used.

Objective of the proposed system is to attain an efficient retrieval of images by combining contour, texture, color, edge and spatial feature for image extraction. As it integrates all these features it could ensure better retrieval efficiency. Image retrieval comprises of two phases namely 1) feature extraction 2) measurement of similarities of query and database images. Applications of the proposed system finds in the fields like crime prevention say for example face recognition by police forces., security check using finger prints, Medical field for the comparison of present diagnosis results with the relevant past cases, Identification of intellectual field such as trade mark recognition, Digital libraries and entertainment purposes etc. This reveals the need for an efficient retrieval system.

\section{PROPOSED SYSTEM ARCHITECTURE}

The proposed system generates feature set incorporating the contour, texture, color, spatial and edge features of all the attainable images in the database for the image retrieval. The attained features are concatenated for the feature set creation of the images. These feature set is compared with query 
feature set for image retrieval. Architecture for the proposed system is shown in Fig. 1

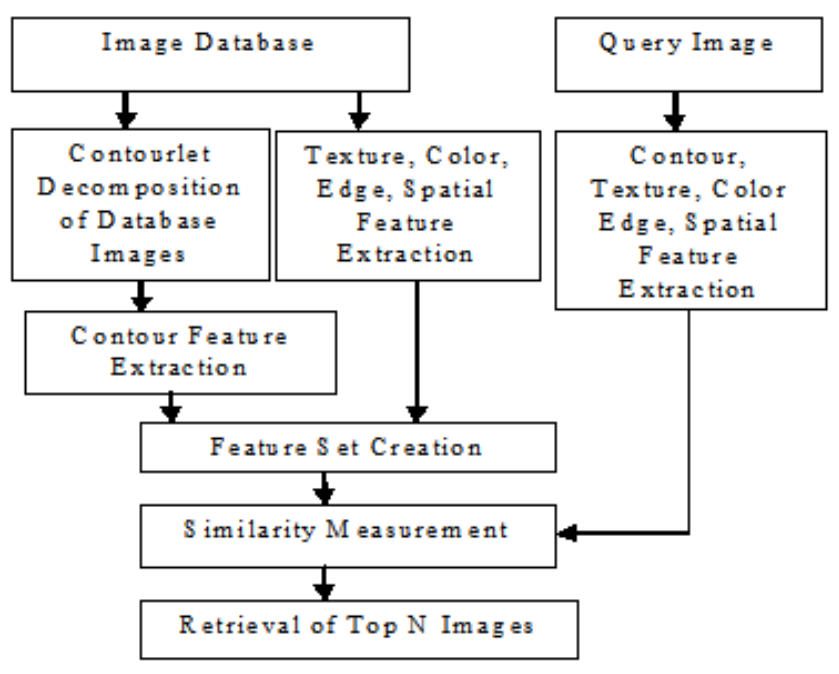

Fig -1: Architecture of proposed system

\section{CONTOUR AND TEXTURE FEATURE}

Prior to the feature extraction all the data images are resized to a unique scale and converted to gray scale images.

\subsection{Contour Feature Extraction}

Resized image is subjected to contourlet decomposition. It constitutes a dual filter bank system. Headmost being a laplacian pyramid (LP) for capturing point discontinuities and followed by $\mathrm{DFB}$ (directional filter bank) for linking point discontinuities.[1] LP decomposition process outputs the low pass version of image and band pass version

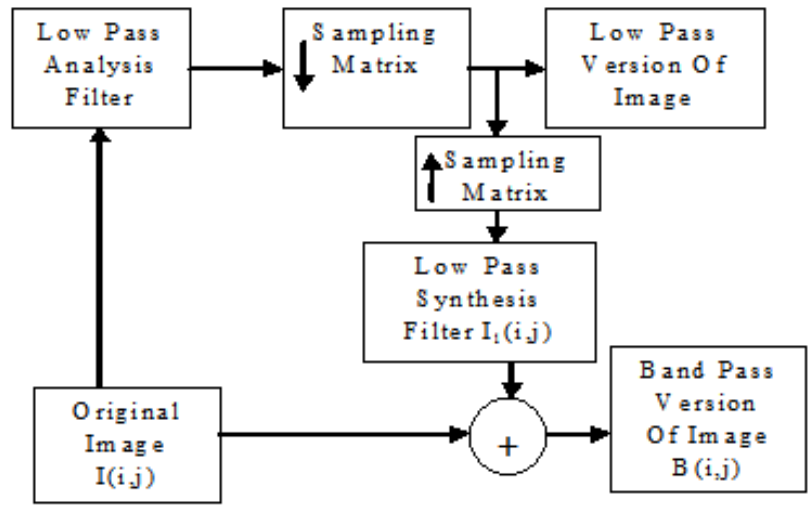

Fig -2: Laplacian pyramid decomposition (one-level)

Band pass version of the image can be calculated as,

$$
B(i, j)=I(i, j)-I_{1}(i, j)
$$

Further pyramidal decomposition [6] is executed on the low pass version of the image. Band pass version is devised to capture smooth contour and directional edges i.e. high frequency contents.DFB is designed based on two building blocks.[1]The first one is a two channel quincunx filter bank with fan filters which divides a two dimensional spectrum in to horizontal and vertical direction. The second one is a shearing operator for reordering image pixels.[8] These two operations preserve directional information. Dual filter bank, results in decomposition of image in to different directional sub band. For the decomposed image in contourlet domain, contour features such as standard deviation $(\sigma s)$ and energy $\left\{\mathrm{E}_{\mathrm{s}}\right\}$ are calculated as per the equations given below.[4]

$$
\begin{aligned}
& \sigma \mathrm{S}=\sqrt{\left(\frac{1}{\mathrm{M} \times \mathrm{N}}\right) \sum_{\mathrm{X}=0}^{\mathrm{M}-1} \sum_{\mathrm{Y}=0}^{\mathrm{N}-1}(\operatorname{Cs}(\mathrm{x}, \mathrm{y})-\mu \mathrm{s})^{2}} \\
& \left\{\mathrm{E}_{\mathrm{s}}\right\}=\left(\frac{1}{\mathrm{M} \times \mathrm{N}}\right) \sum_{\mathrm{x}=0}^{\mathrm{M}-1} \sum_{\mathrm{Y}=0}^{\mathrm{N}-1}(|\mathrm{Cs}(\mathrm{x}, \mathrm{y})|)
\end{aligned}
$$

Where Cs is coefficient of CT (contourlet transform) decomposed $s^{\text {th }}$ sub band. $\mu$ s being mean value of $s^{\text {th }} s u b$ band. $\mathrm{M} \times \mathrm{N}$ represents size of CT decomposed sub band. Energy and standard deviation for all images in database are found. After which Normalization of these two quantities were done and stored as their individual contour feature set.

\subsection{Texture Feature Extraction}

For texture feature extraction rotation invariant local binary pattern (LBP) histogram Fourier features [2] based on uniform LBP histogram is used. LBP is a powerful operator.LBP for the central pixel $(\mathrm{x}, \mathrm{y})$ of image $\mathrm{I}(\mathrm{x}, \mathrm{y})$ is obtained as,

$$
\operatorname{LBP}_{\mathrm{SR}}(\mathrm{x}, \mathrm{y})=\sum_{S=0}^{\mathrm{S}-1}\left(\mathrm { T } \left(\mathrm{I}(\mathrm{x}, \mathrm{y})-\mathrm{I}\left(\mathrm{x}_{\left.\mathrm{s}, \mathrm{y}_{\mathrm{s}}\right)}\right) 2^{\mathrm{s}}\right.\right.
$$

$\mathrm{T}(\mathrm{z})$ - Threshold function, $\mathrm{s}-$ sampling point ,r- radius. $\mathrm{T}(\mathrm{z})$ is given by,

$$
\mathrm{T}(\mathrm{z})=\left\{\begin{array}{l}
1, \mathrm{z} \geq 0 \\
0, \mathrm{z}<0
\end{array}\right.
$$

LBP is considered as uniform, if its binary pattern contains only two bitwise transitions.

Steps involved for texture feature extraction are,

1. Conversion of database images in to gray scale.

2. Computation of LBP.

3. Generation of histogram of LBP.

4. Computation of Discrete Fourier transform of the histograms per the equation,

$$
\mathrm{H}(\mathrm{n}, \mathrm{u})=\sum_{\mathrm{r}=0}^{\mathrm{p}-1} \mathrm{~h}_{1} \mathrm{u}_{\mathrm{s}}(\mathrm{n}, \mathrm{r}) \mathrm{e}^{-\mathrm{j} 2 \Pi n \mathrm{r} / \mathrm{s}}
$$

5. Store the absolute value of obtained DFT as feature set.

For the image and the rotated version by an angle, the absolute value of DFT of histogram remains entirely the same. 


\section{COLOR, EDGE AND SPATIAL FEATURE}

For color extraction histogram based approach is employed. Canny's edge detection technique is used for edge capturing. Spatial feature based on dominant color extraction provides the details of spatial feature.

\subsection{Color Extraction}

For color extraction, red, green and blue color values are extracted.[5] Generation of respective histograms and computation of average red, green and blue content is performed. Result is stored in a 1D matrix and stored as color feature set. Color moments such as mean, variance and skewness were found to be effective for analyzing current distribution of the image. Mean $\mu_{\mathrm{x}}$, Variance $\sigma_{\mathrm{x}}$, Skewnesss $_{\mathrm{x}}$, obtained as,[11]

$$
\begin{aligned}
\mu_{x} & =\frac{1}{N} \sum_{y=1}^{N} f_{x y} \\
\sigma_{x} & =\left(\frac{1}{N} \sum_{y=1}^{N}\left(f_{x y}-\mu_{x}\right)^{2}\right)^{1 / 2} \\
s_{x} & =\left(\frac{1}{N} \sum_{y=1}^{N}\left(f_{x y}-\mu_{x}\right)^{3}\right)^{1 / 3}
\end{aligned}
$$

$f_{x y}-x^{\text {th }}$ color component of the $y^{\text {th }}$ image pixel's represents the total number of pixels in an image. Mean, Variance and Skewness were also concatenated to the color feature set.

\subsection{Edge Extraction}

For the edge feature set creation, Canny's edge detection technique is preferred. Canny edge detector employs a multi stage algorithm for its edge detection. Good detection, localization and minimal response make it suitable for edge detection. The algorithm [3] employed for edge feature capture is as follows,

1. Input image and scale it to required scale.

2. Conversion of resized image to gray scale one.

3. Detection of edge using Canny's edge detection and store in to a two dimensional matrix.

4. Maximum value of each column is extracted from the obtained result and stored as the edge feature set.

\subsection{Spatial Feature Extraction}

Spatial feature based on dominant color extraction is performed. In order to procure spatial features, each image in database is initially transformed in to NTSC color system. It includes red, green, blue, magenta, white, yellow, cyan, aquamarine, black, gray, dark red and copper. After which image $\mathrm{I}$ is divided to $\mathrm{M}$ sub regions. For each sub regions occurrence count is extracted. The color count for each sub region and indices are given by [4],

$$
\{\text { Nocc }\}_{x}=\left\{\{\text { Socc }\}_{0}{ }^{(x)},\{\operatorname{Socc}\}_{1}^{(x)}, \ldots \ldots\{\text { Socc }\}_{N s}{ }^{(x)}\right\}
$$

Where $\{\text { Socc }\}_{\mathrm{v}}{ }^{(\mathrm{x})}$ is a subset of $\{\text { Mocc }\}^{(\mathrm{x})}$. The subset of \{Mocc\}are sorted such that subset with maximum valued element is listed as the first subset and remaining trailing it. The index of the first $M_{\text {sf }}$ sub regions is determined and corresponding indices are gathered as spatial feature set.

After abstraction of aforesaid feature set (contour, texture, color, edge, spatial) from image database, the individual feature sets are concatenated in to single feature set. Normalization [4] of the feature set is carried as per the equation,

Where

$$
\{\mathrm{F} 1 \mathrm{Sx}\}=(\sqrt[2]{\mathrm{p} / \mathrm{q}}) \times \mathrm{FSx}^{\prime}(\mathrm{r})
$$

$$
\begin{aligned}
\mathrm{p} & =\left|\mathrm{FSx}^{\prime}\right| \\
\mathrm{q} & =\sum_{\mathrm{r}=0}^{\mathrm{p}-1}\left(\mathrm{FSx}^{\prime}(\mathrm{r})\right)^{2} \\
\mathrm{FSx}^{\prime}(\mathrm{r}) & =\mathrm{FSx}(\mathrm{r})|\mathrm{FSx}|-\sum_{\mathrm{r}=0}^{|\mathrm{FSx}|-1}(\mathrm{FSx}(\mathrm{r}))
\end{aligned}
$$

The Normalized feature set is obtained from (11).It is the definite feature set extracted for the particular image in the database. The obtained result is stored as the feature database.

\section{QUERY BASED IMAGE RETRIEVAL}

When query image is given as input, system computes the feature set for the same. Then determined feature set is compared with feature set in the feature database using available similarity measure techniques such as Squared Euclidian Distance (SED), Manhattan Distance etc.

\subsection{Similarity Measures}

The SED computes similarity between feature database $\left\{\mathrm{F} 1 \mathrm{~S}_{\mathrm{x}}(\mathrm{r})\right\}$ and the feature set of the query image $\mathrm{FS}_{\mathrm{qu}}(\mathrm{r})$.SED can be calculated [4] by the equation (15)

$$
\mathrm{D}_{\mathrm{sed}}=\sum_{\mathrm{r}=0}^{|\mathrm{FSx} \prime|-1}(\mathrm{~F} 1 \mathrm{Sx}(\mathrm{r})-\mathrm{FSqu}(\mathrm{r}))^{2}
$$

Another similarity measure technique called Manhattan gives the sum of absolute difference distance measure.

$$
\mathrm{D}_{\mathrm{M}}=\sum_{\mathrm{r}=0}^{|\mathrm{FSx} \prime|-1} \mid\left(\mathrm{F} 1 \mathrm{Sx} \mathrm{x}^{\prime}(\mathrm{r})-\mathrm{FSqu}(\mathrm{r}) \mid\right.
$$

The images that accure least SED/ Manhattan value are sorted such that they obtain the first location and top $\mathrm{N}$ matches are retrieved.

\section{RESULTS AND DISCUSSIONS}

In the proposed system, initially contourlet decomposition (CD) is performed for the contour feature extraction. All the images in the database are decomposed. A sample image from database and its corresponding $\mathrm{CD}$ are shown in Fig 3. Four level LP decomposition is performed using pkva filter 
[1].Standard deviation and energy [4] are used as contour feature, which is computed on each direction sub band of the decomposed images in the database. Normalized result was used for the creation of contour feature set.

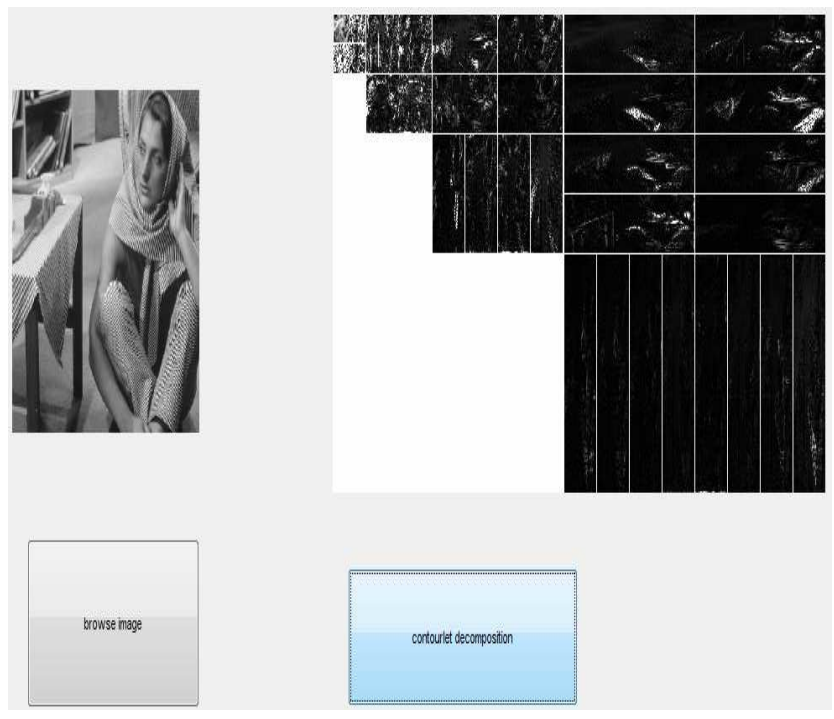

Fig -3: Contourlet transform decomposed image

After the creation of contour feature set, we have extracted texture feature using rotation invariant LBP histogram Fourier features [2].For this we have computed LBP for all database images and have generated corresponding histogram of the obtained LBP's. Followed by this computation of absolute value of the DFT of generated histogram was performed. It was used for the creation of texture feature set. A sample image from database, its histogram, LBP and histogram of LBP are shown in Fig 4.

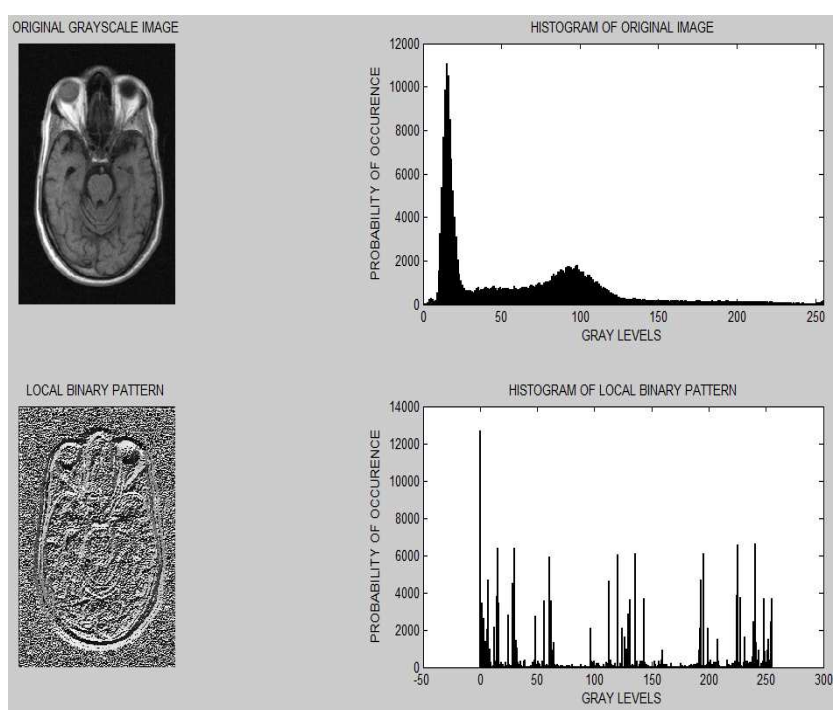

Fig -4: Local binary pattern and histogram
Original image and its rotated version by an angle $270^{\circ}$ are illustrated by Fig. 5 and Fig.6 respectively.

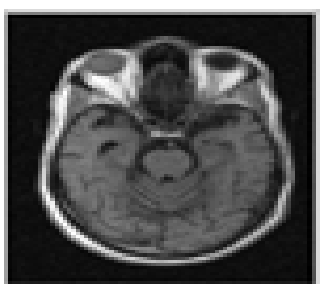

Fig -5: Input image

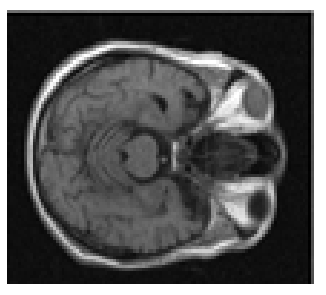

Fig -6: Input image rotated by an angle $270^{\circ}$

For the rotated version of same input image, absolute value of DFT of histograms remains same as that of original version, although LBP contains a two bitwise transitions.LBP of the same image and its rotated version are exhibited in Table1.

Table -1: Local binary pattern

\begin{tabular}{|c|c|}
\hline Image & LBP \\
\hline LBP of input image & 00000110 \\
\hline $\begin{array}{c}\text { LBP of input image rotated } \\
\text { by an angle } 270^{\circ}\end{array}$ & 01110000 \\
\hline
\end{tabular}

The absolute value plot of DFT of the generated histogram for the original and rotated versions are displayed in Fig 7

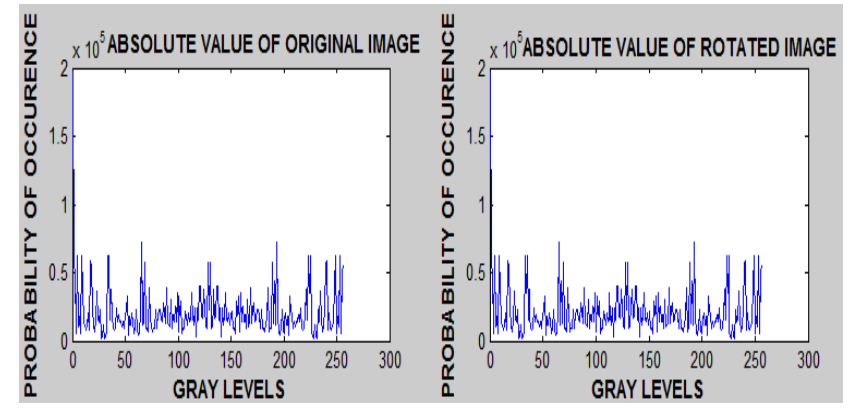

Fig -7: Absolute value of input image and $270^{\circ}$ rotated version of the same input image

After the formation of texture feature set, we have created color feature set. For these individual mean values of red, green and blue were extracted for all database images. Results 
obtained were stored as color feature set. A sample image from database and corresponding RGB histogram is shown in Fig 8.

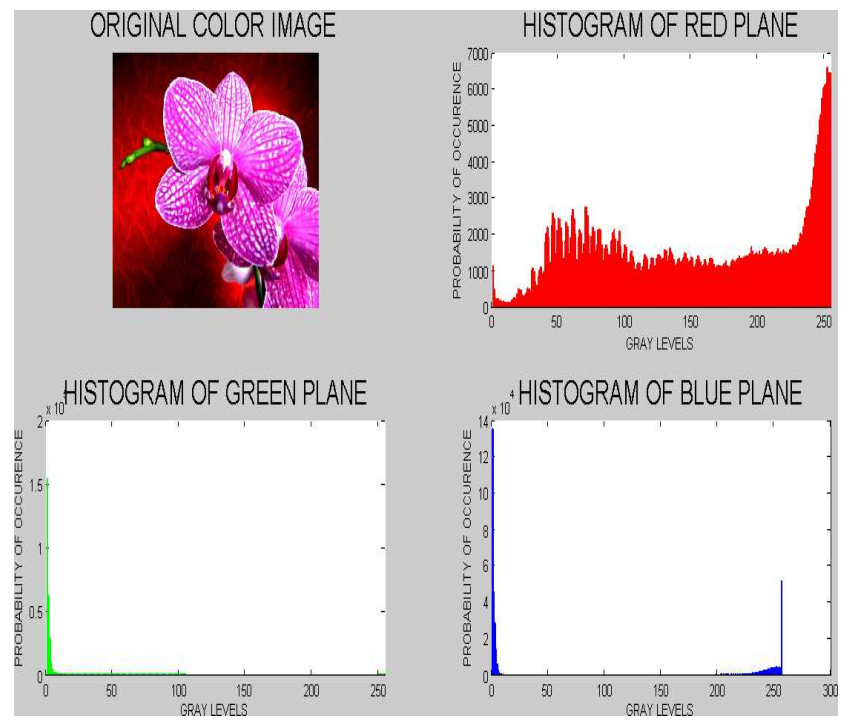

Fig -8: Histogram of Red, Green, Blue plane.

Table 2 illustrates the mean value generated for the selected sample image.

Table -2: Color components

\begin{tabular}{|c|l|}
\hline Color & Mean value \\
\hline Blue & 101.3969 \\
\hline Green & 47.0693 \\
\hline Red & 164.2397 \\
\hline
\end{tabular}

Color feature set creation was followed by edge feature set extraction. Canny's edge detection was employed for detecting edges of all the available database images.Fig.9 shows a sample database image and its edge detected image.

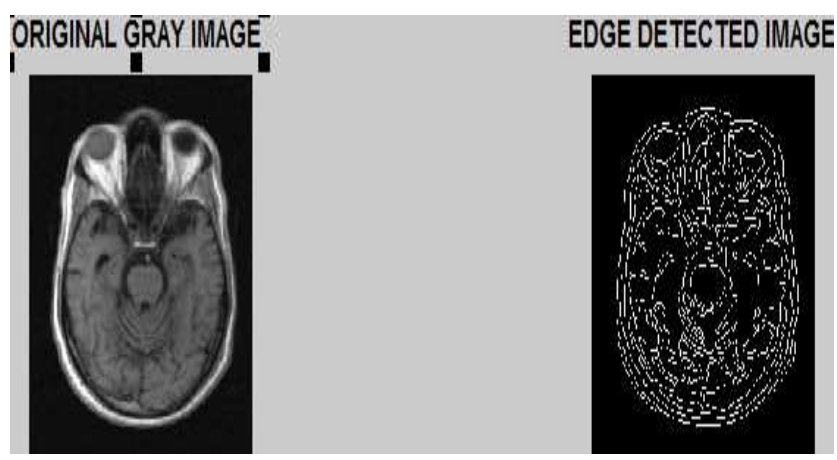

Fig -9: Edge detection.
Final step in the feature set creation was spatial feature set extraction. Here we have extracted dominant color from the sub regions of all the database images. NTSC color system was used. Fig 10 displays a sample image in NTSC color system and Table 3 illustrates its obtained color components.

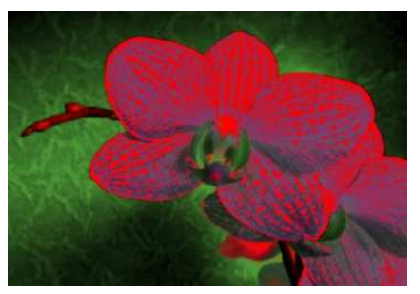

Fig -10: Sample image (NTSC Color system)

Table -3: Color components NTSC color system

\begin{tabular}{|c|c|}
\hline Color & Mean value \\
\hline White & 0.7151 \\
\hline Black & 0 \\
\hline Blue & 0.1635 \\
\hline Copper & 0.5389 \\
\hline Cyan & 0.3689 \\
\hline Dark Red & 0.1731 \\
\hline Gray & 0.3575 \\
\hline Green & 0.2053 \\
\hline Magenta & 0.5098 \\
\hline Red & 0.3461 \\
\hline Yellow & 0.5516 \\
\hline Aquamarine & 0.5462 \\
\hline
\end{tabular}

All the foresaid features were concatenated in to a single feature set and normalization was performed to create feature set of the database image .Based on the feature set of database images and query image SED and Manhattan distance were calculated. Fig 11 illustrates minimum SED/Manhattan distance plot (here 35 images are considered to create the database).

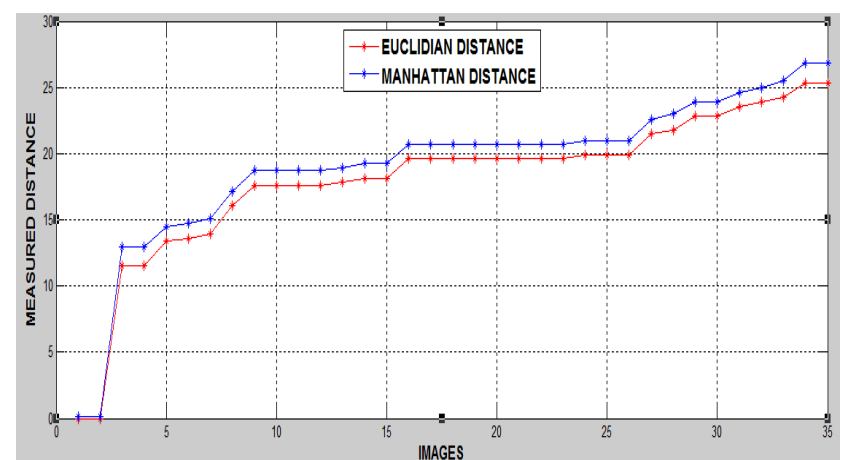

Fig -11: Minimum distance plot 
Finally, images were sorted based on the minimum SED/Manhattan distance and retrieval of top $\mathrm{N}$ images was performed. Fig 12 displays a sample Query image and corresponding retrieved images.

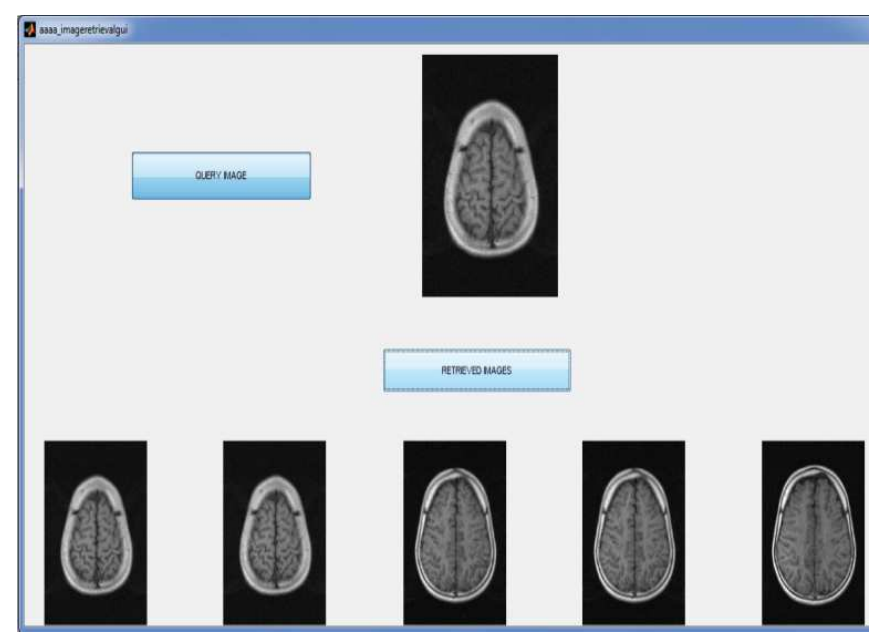

Fig -12: Image retrieval for a query image.

\subsection{Performance Evaluation}

Performance evaluation of the proposed system was carried out by computing precision-recall rate, error rate and traditional $\mathrm{F}$ measure, as per the equations given below,

$$
\begin{gathered}
\text { Recall rate }(\mathrm{R})=\frac{\text { No:of relevant images retrieved }}{\text { Total no: of relevant images in database }} \\
\text { Precision rate }(\mathrm{P})=\frac{\text { No: of relevant images retrieved }}{\text { Total no:of images in database }} \\
\text { Error rate }=\frac{\text { No: of non relevant images retrieved }}{\text { Total no:of images in database }} \\
\text { F measure }=\frac{2(\mathrm{P} \times \mathrm{R})}{(\mathrm{P}+\mathrm{R})}
\end{gathered}
$$

The figure shown below indicates the obtained rates for different query images.

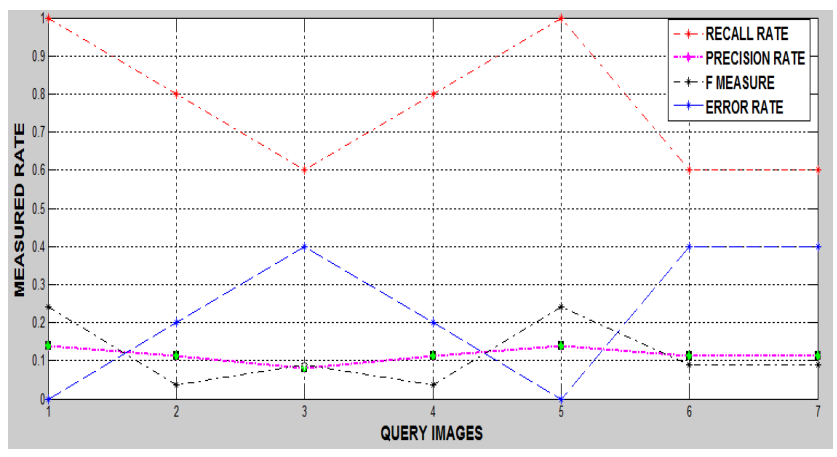

Fig -13: Rate plot for different query image.
Precision and recall are the standard measures in the image retrieval system which provides a good indication of the system performance [9]. Recall can be made one by retrieving all images and precision can be kept high by retrieving only few relevant images. Reduction in the recovery of non relevant images will keep the error rate minimum. The weighted harmonic mean of precision and recall is obtained via $\mathrm{F}$ measure.

\section{CONCLUSIONS}

In this paper, an efficient image retrieval system based on contourlet, texture, spatial, edge , and color features were proposed. CT helps to extract directional contours, horizontal and vertical edges. The similarity measure techniques such as SED, Manhattan were found effective for image retrieval. Experimental results and performance evaluation shows that our proposed system offers a better image retrieval compared to the traditional ones which use single features for image retrieval. Further extension of proposed work can be carried out by comparing various similarity measure techniques such as Minkowsi-form distance, Quadratic form distance, Mahalanobis distance, Kullback-leiber divergence and Jeffery - divergence etc.

\section{REFERENCES}

[1]. Ch.SrinivasaRao , S. Srinivaskumar, B.N.Chatterji, "Content Based Image Retrieval using Contourlet Transform", International Journal on Graphics, Vision and Image Processing, Vol. 7, No. 3, pp. 9-15, 2007.

[2]. TimoAhonen, Jiri Matas, Chu He and MattiPietik"ainen "Rotation Invariant Image Description with Local Binary Pattern Histogram Fourier Features" in Proceedings of 16th Scandinavian Conference on Image Analysis, pp. 61 - 70, June 15 - July 18, Oslo, Norway, 2009

[3]. PoulamiHaldar,Joydeep Mukherjee "content based image retrieval using Histogram, Color and Edge"International journal of computer applications,june 2012

[4]. B.syam and Y.srinivasaRaoIntegrating contourlet features with texture,color and spatial features for effective image retrieval" [IEEE],2010

[5]. Rahul Mehta,Nischol Mishra, Sanjeev Sharma "colortexture based image retrieval system"International journal for computer applications, june 2011

[6]. "Ch.SrinivasaRao and S.Srinivas Kumar, "Content Based Image Retrieval using Contourlet Sub-band Decomposition", Proceedings of SPIT-IEEE Colloquium and International Conference, Vol. 1, Mumbai, India

[7]. K.S.S. Prakash and R.M.D. Sundaram, "Combining Novel features for Content Based Image Retrieval,"in proceedings of 14th IEEE Workshop on Multimedia Communications and Services,

[8]. Minh N. Do and Martin Vetterli "Pyramidal directional filter banks and curvelets", in IEEE international conference on image processing,Thessaloniki,Greece,2001 
[9]. Henning Muller, Wolfgand, David, Stephane, and Thietty "Performance evaluation in CBIR, over view and proposals" computer vision group.

[10]. B.G Prasad,S.K Gupta and K.K.Biwas,"color and shape index for region based image retrieval"in proceedings of the $4^{\text {th }}$ international workshop on visual form.

[11]. NeetuSharma.S,PareshRawat.S and JaikaranSingh.S

"Efficient CBIR using color histogram processing”,SIPIJ,2011 